\title{
Construction and characterization of a eukaryotic expression vector for small interfering RNA targeting the NEDD9 gene
}

\author{
JING-XIA CHANG ${ }^{1}$, HUA-QI WANG ${ }^{1}$, GUO-QIANG ZHAO ${ }^{2}$, HE-YING CHU $^{1}$ and GUO-JUN ZHANG ${ }^{1}$ \\ ${ }^{1}$ Department of Respiratory Medicine, The First Affiliated Hospital of Zhengzhou University, Zhengzhou, Henan; \\ ${ }^{2}$ Department of Microorganisms and Immunization, Preclinical Medicine, Zhengzhou University, \\ Zhengzhou, Henan, P.R. China
}

Received June 19, 2012; Accepted September 3, 2012

DOI: $10.3892 /$ ijmm.2012.1137

\begin{abstract}
The aim of this study was to construct a eukaryotic expression vector for a small interfering RNA (siRNA) targeting the neural precursor cell expressed, developmentally downregulated 9 (NEDD9) gene, and to investigate the effects of RNA interference (RNAi) on NEDD9 expression in human lung adenocarcinoma A549 cells. We used the siRNA design and analysis software to determine the target oligonucleotides according to the sequence of NEDD9 mRNA available in GenBank. Four siRNA sequences were obtained, and the corresponding cDNAs were synthesized and inserted into the pRNAT-CMV3.2 plasmid to construct the recombinant plasmids. These were transformed into the $E$. coli strain DH5 $\alpha$. The plasmids, after identification by PCR and DNA sequencing, were transfected into the A549 cell line via the liposome method. NEDD9 mRNA and protein in the cells were determined by fluorescence quantitative RT-PCR (FQ-PCR) and western blotting, respectively. The pRNAT-CMV3.2transfected plasmid was used as a control. Four recombinant plasmids were identified by PCR and sequence analysis, which contained the correct insertion of the designed sequences in the plasmids. FQ-PCR and western blotting showed substantially decreased mRNA and protein expression of the NEDD9 gene in the transfected cells, compared with the control group. In conclusion, the recombinant plasmids expressing the siRNA targeting the NEDD9 gene were successfully constructed, and the siRNA expression vectors inhibited the expression of NEDD9 in A549 cells.
\end{abstract}

\section{Introduction}

The neural precursor cell expressed, developmentally downregulated 9 (NEDD9), also known as HEF1 and Cas-L

Correspondence to: Professor Guo-Jun Zhang, Department of Respiratory Medicine, The First Affiliated Hospital of Zhengzhou University, 1 Jianshe East Road, Zhongyuan, Henan 450052, P.R. China E-mail: guojunzh001@yahoo.com.cn

Key words: lung adenocarcinoma, developmentally downregulated 9, RNA interference, A549 cells
$(1,2)$, acts as a scaffold protein and belongs to the family of Crk-associated substrate (CAS) proteins that regulate protein complexes controlling cell attachment, migration, invasion, cell cycle, apoptosis, and oncogenic signal transduction (3). Overexpression of the NEDD9 protein has now been strongly linked to poor prognosis in cancer, as well as resistance to first-line chemotherapeutics in multiple tumor types including breast cancer (4), glioblastoma (5) and melanoma (6). Lung cancer is still a serious health problem and remains the first most common type of fatal cancer worldwide (7). High levels of NEDD9 mRNA and protein have been shown to be present in human lung adenocarcinoma tissues (8). In cell lines, NEDD9 has been identified as a metastasis-promoting gene (9).

Small interfering RNA (siRNA) are short pieces of doublestranded RNA (19-22 nt). The molecule works by cleaving and destroying its cognate RNA. siRNA first assembles into RNA-induced silencing complexes (RISCs), where it activates the complex by unwinding its RNA strands. The unwound RNA strands subsequently guide the complex to the complementary RNA molecules, where the complex cleaves and destroys the cognate RNA, resulting in the RNA interference (RNAi) phenomenon. RNAi has evolved into a powerful tool to study gene functions and it also has potential applications in gene therapy. RNAi has evolved into a powerful molecular biological tool to study gene functions $(10,11)$, with potential applications in gene therapy.

The current study constructed and screened for short hairpin RNA (shRNA) in response to the NEDD9 targeting gene. The NEDD9 siRNA was transfected into the human lung adenocarcinoma cell line A549 with Lipofectamine 2000. The cells were transfected with siRNA and the untreated cells were used as controls. The inhibitory effect of siRNA on the expression of NEDD9 mRNA and protein was detected by fluorescence quantitative RT-PCR (FQ-PCR) and western blotting.

\section{Materials and methods}

Reagents and instruments. The pRNAT-CMV3.2 plasmid was purchased from GenScript Co. (USA). E. coli DH5 $\alpha$ and restriction enzymes were obtained from the Department of Microorganism and Immunization, Preclinical Medicine, 
Zhengzhou University (China). T4 DNA ligase and marker DL2000 were purchased from Takara Bio, Inc. (Japan). Agarose was purchased from Oxoid (UK). The plasmid DNA mini-preparation, the DNA gel extraction and PCR kits were purchased from Axygen (USA). The inverted fluorescence microscope was from Olympus Corporation (Japan).

Cell culture. The human lung adenocarcinoma cell line A549 was a gift from the Sino-British Research Centre for Molecular Oncology of Zhengzhou University. A549 cells were cultured in EMDM medium (Hyclone, Logan, UT, USA) supplemented with $10 \%$ fetal bovine serum (FBS) (Hyclone), $100 \mathrm{IU} / \mathrm{ml}$ penicillin and $100 \mu \mathrm{g} / \mathrm{ml}$ streptomycin (Hyclone). Cells were maintained at $37^{\circ} \mathrm{C}$ in a humidified chamber containing $5 \%$ $\mathrm{CO}_{2}$.

siRNA design. According to the principles of siRNA design and the Homo sapien NEDD9 gene sequence (GenBank accession no. NM_182966), three pairs of specific siRNA sequences GCTGCCGAAATGAAGTATA (159-117), GGGCCTTATAT GACAATGT (193-211), GTGTCCTATTTCTTAGTGA (648666) and the negative control siRNA vector sequence TTCTC CGAACGTCGCACGT (CO) were synthesized using software provided at http://www.mpibpc.gwdg.de/abteilungen/100/105/ sirna.html. These siRNA sequences were composed of a sense strand, an antisense strand, reverse complementary sequences and a loop. A TTTTT termination signal was introduced to the 3'-end of the oligos. BamHI and $\mathrm{XhoI}$ restriction enzyme digestion sites were also designed in shRNA for further cloning. The eight oligonucleotides were synthesized by Shanghai BioSune Biotechnology Co. (Shanghai, China) (Table I).

Annealing and purification of specific target sequences. The four pairs of synthesized oligonucleotides (N159F and N159R, N193F and N193R, N648F and N648R, COF and COR) were dissolved in $50 \mu \mathrm{l}$ ultrapure water. From these stocks, $13.5 \mu \mathrm{l}$ of each oligonucleotide pair was mixed with $3 \mu \mathrm{l}$ of $10 \mathrm{X}$ buffer. The annealing step was performed by a 5 -min incubation at $95^{\circ} \mathrm{C}$, followed by a $10 \mathrm{~min}$ incubation at $70^{\circ} \mathrm{C}$. The annealed oligos were then slowly cooled to $37^{\circ} \mathrm{C}$. The annealed NEDD9 and short hairpin DNA control oligos were loaded onto 2.0\% agarose gels for separation and purification of the vector.

Annealed products ligated into the pRNAT-CMV3.2 vector. DNA ligation was performed by incubating $10 \mu \mathrm{l}$ of the reaction solution containing $3 \mu \mathrm{l}$ of the annealed oligonucleotides, $1 \mu 1$ linear pRNAT-CMV3.2 after BamHI and XhoI enzyme double digestion, $1 \mu 1$ of $10 \mathrm{X}$ T4 buffer and $1 \mu 1 \mathrm{~T} 4$ DNA ligase and incubated at $16^{\circ} \mathrm{C}$ overnight. The recombinant vector was transformed into DH5 $\alpha$ competent cells. Positive clones of vector pRNAT-CMV3.2-N159, pRNAT-CMV3.2N193, pRNAT-CMV3.2-N648 and pRNAT-CMV3.2-C were randomly selected and inoculated into $30 \mathrm{ml}$ of LB medium supplemented with ampicillin sodium. After the cells were cultured in a shaking incubator overnight $(16-18 \mathrm{~h})$ at $37^{\circ} \mathrm{C}$, the DNA plasmids were extracted using a plasmid DNA mini-preparation kit according to the manufacturer's instructions, and identified by $1 \%$ agarose gel electrophoresis after restriction enzyme digestion. DNA Marker 2000 was used to estimate the length of the fragments. The confirmed positive clones producing the four vectors were randomly selected and inoculated into $30 \mathrm{ml} \mathrm{LB}$ medium supplemented with ampicillin sodium. After the cells were cultured in a shaking incubator overnight $(16-18 \mathrm{~h})$ at $37^{\circ} \mathrm{C}, 1 \mathrm{ml}$ of medium was used for sequencing.

Plasmid transfection into the A549 cell line. Liposomes were used to facilitate transfection. Metafectene liposomes and the plasmid were mixed in a ratio of 3:1 (12). The culture medium containing serum and antibiotic was removed prior to transfection and washed three times with PBS, followed by the addition of culture medium but without serum and antibiotics. After $1 \mathrm{~h}$, the A549 cells were transfected (when the cells reached $80-90 \%$ ) by placing the mixture in 6 -well culture plates and adding $0.8 \mathrm{ml}$ of culture medium without serum; the plates were incubated at $37^{\circ} \mathrm{C}$ for $6-8 \mathrm{~h}$. After incubation, $2 \mathrm{ml}$ of the culture medium with $20 \%$ FBS was added in $5 \% \mathrm{CO}_{2}$ and cultured for a further $48 \mathrm{~h}$ at $37^{\circ} \mathrm{C}$. Transfection efficiency was observed using an inverted fluorescence microscope as the vector carries the coral green fluorescent protein (cGFP) as a marker.

RNA isolation and establishment of a method of FQ-PCR detection of NEDD 9 mRNA. Cultured A549 cells were divided into six groups and either transfected with the three vector plasmids pRNAT-CMV3.2-N159, pRNAT-CMV3.2-N193 and pRNAT-CMV3.2-N648, transfected with an empty vector plasmid, transfected with a plasmid containing an unrelated pRNAT-CMV3.2-C sequence, or no plasmid. The relative rate of NEDD9 mRNA expression in these six groups was analyzed via FQ-PCR, and the rate of suppression of mRNA expression was calculated.

Total RNA was purified from cells using a TRIzol reagent (Invitrogen, Carlsbad, CA, USA). The first-strand of the cDNA was synthesized using $2.5 \mu \mathrm{g}$ RNA and AMV retroviridase (Promega). Specific primers were designed in accordance with the mRNA sequence of the NEDD9 gene and the NEDD9 and GAPDH segments were amplified. The primers were provided by Shanghai Bioengineering Co. Primers for the NEDD9 gene were upstream, 5'-CGTGGGTAAAAAGGTGTTCC-3' and downstream, 5'-CAAGCCTCCAAACTCAGGAC-3' (amplified segment $124 \mathrm{bp}$ ); primers for GAPDH were upstream, 5'-TCGTGG AAGGACTCATGACC-3' and downstream, 5'-AGGGATGA TGTTCTGGAGAG-3' (amplified segment $97 \mathrm{bp})$.

NEDD9 mRNA levels were quantified by FQ-PCR based on TaqMan ${ }^{\mathrm{TM}}$ technology, using the ABI PRISM 7500 Sequence Detection System (Applied Biosystems, Foster City, CA, USA). The reaction system consisted of $20 \mu 12 \mathrm{X}$ real-time PCR buffer, $0.5 \mu \mathrm{l}$ of the upstream and downstream primers of NEDD9, $2 \mu 1$ reverse transcription product, $0.2 \mu 1$ TaqDNA polymerase, and $\mathrm{ddH}_{2} \mathrm{O}$ to increase the final volume to $40 \mu \mathrm{l}$. FQ-PCR reaction conditions included pre-denaturation at $95^{\circ} \mathrm{C}$ for $3 \mathrm{~min} ; 95^{\circ} \mathrm{C}$ for $15 \mathrm{sec}, 65^{\circ} \mathrm{C}$ for $45 \mathrm{sec}, 40$ cycles; $72^{\circ} \mathrm{C}$ for $2 \mathrm{~min}$. GAPDH was simultaneously amplified as an internal reference. All reactions were performed in triplicate. The results were analyzed by calculating the $\mathrm{Ct}$ values for NEDD9 and GAPDH in the samples and the relative expression of NEDD9 mRNA in each group [the relative fold (RF)] using $2^{-\Delta \Delta \mathrm{Ct}}$ value was calculated. 
Table I. Designed oligonucleotide sequences targeting the NEDD9 gene.

\begin{tabular}{ll}
\hline NEDD9 & \multicolumn{1}{c}{ Sequences $\left(5^{\prime} \rightarrow 3^{\prime}\right)$} \\
\hline N159F & 5'-GATCCGCTGCCGAAATGAAGTATATTCAAGAGATATACTTCATTTCGGCAGCTTTTTTC-3' \\
N159R & 5'-TCGAGAAAAAAGCTGCCGAAATGAAGTATATCTCTTGAATATACTTCATTTCGGCAGCG-3' \\
N193F & 5'-GATCCGGGCCTTATATGACAATGTTTCAAGAGAACATTGTCATATAAGGCCCTTTTTTC-3' \\
N193R & 5'-TCGAGAAAAAAGGGGCCTTATATGACAATGTTCTCTTGAAACATTGTCATATAAGGCCCG-3' \\
N648F & 5'-GATCCGTGTCCTATTTCTTAGTGATTCAAGAGATCACTAAGAAATAGGACACTTTTTTC-3' \\
N648R & 5'-TCGAGAAAAAAGTGTCCTATTTCTTAGTGATCTCTTGAATCACTAAGAAATAGGACACG-3' \\
COF & 5'-GATCCTTCTCCGAACGTCGCACGTTTCAAGAGAACGTGACACGTTCGGAGAATTTTTC-3' \\
COR & 5'-TCGAGAAAAAATTCTCCGAACGTCGCACGTTCTCTTGAAACGTGACACGTTCGGAGAAG-3'
\end{tabular}

NEDD9, neural precursor cell expressed, developmentally downregulated 9. The bold letters represent the target sequences.

Protein isolation and western blot analysis. NEDD9 expression was examined in A549 cells transfected with plasmid vectors using western blotting. Cells were harvested in lysis buffer (2\% SDS, $50 \mathrm{mM}$ Tris, pH 7.4/1 mM EDTA/protease inhibitor mixture) $48 \mathrm{~h}$ after transfection and homogenized by sonication. Their protein concentrations were determined using a DTX 880 Multimode Detector (Beckman Coulter, Fullerton, CO, USA). Equal amounts of protein $(40 \mu \mathrm{g})$ were separated by $10 \%$ sodium dodecyl sulfate-polyacrylamide gel electrophoresis (SDS-PAGE) on $8 \%$ gels and were transferred onto nitrocellulose membranes (Hyclone). The membranes were blocked with $5 \%(\mathrm{v} / \mathrm{v})$ skimmed milk and probed with phospho rabbit-anti-human polyclonal primary antibodies for NEDD9 (93 kDa) (Abcam, San Francisco, CA, USA), at $4^{\circ} \mathrm{C}$ overnight. The membranes were washed and incubated with horseradish peroxidase (HRP)-conjugated goat-antirabbit secondary antibody (Beijing Zhong Shan Golden Bridge Biological Technology), at room temperature for $1 \mathrm{~h}$. Antibodies against $\beta$-actin (Santa Cruz Biotechnology, Inc., Santa Cruz, CA, USA) were used to measure protein loading. The bound antibodies were visualized using an electrochemiluminescence system (Amersham Pharmacia Biotech, Buckinghamshire, UK).

Statistical analysis. The SPSS 17.0 (SPSS Inc., USA) for Windows statistical software package was used for analysis. One-way analysis of variance (ANOVA) was used to investigate the differences in NEDD9 mRNA expression among the groups in vitro. A P-value $<0.05$ indicated a significant difference, and $\mathrm{P}$-value $<0.01$ indicated a highly significant difference.

\section{Results}

Annealed oligonucleotides. After annealing the single-strand oligonucleotides (N159, N193, N648, CO) they were visualized by gel electrophoresis. As expected, bright bands with a length of $59 \mathrm{bp}$ were observed (Fig. 1).

Identification of recombinant pRNAT-CMV3.2-siRNA by electrophoresis. Positive clones were selected for plasmid mini-preparation, and the products were digested using BamHI and XhoI enzymes, forming linear DNA fragments

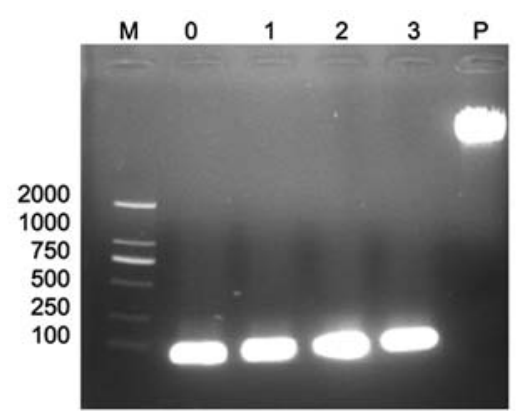

Figure 1. Electrophoresis of the siRNA hairpin DNA after annealing. M, DNA marker; lane 0, product of unrelated siRNA hairpin DNA (CO) after annealing; lane 1, product of NE159 siRNA hairpin DNA after annealing; lane 2, product of NE193 siRNA hairpin DNA after annealing; lane 3, product of NE648 siRNA hairpin DNA after annealing; P, empty vector plasmid.

(Fig. 2A). Fragments were inserted into the pRNAT-CMV3.2 vector and $5 \mu \mathrm{l}$ of the recombinant pRNAT-CMV3.2-siRNA plasmid was visualized by $1 \%$ agar gel electrophoresis. The PCR product of interest is $221 \mathrm{bp}$ in length as it contains the pRNAT-CMV3.2 insert, the forward and reverse primers, and length of the hairpin DNA fragment. All three pairs of specific NEDD9 and the unrelated control recombinant clones are shown in Fig. 2B.

Identification of recombinant pRNAT-CMV3.2-siRNA by DNA sequencing. The pRNAT-CMV3.2-siRNA cell culture was analyzed by DNA sequencing. The sequence was in agreement with the GenBank database (Fig. 3).

Results of the transfection of cells. The three recombinant pRNAT-CMV3.2-siRNA groups (transfected with pRNAT-CMV3.2-NE159, pRNAT-CMV3.2-NE193, pRNATCMV3.2-NE648), the unrelated siRNA group (transfected with pRNAT-CMV3.2-C), and the empty vector plasmid group (transfected with pRNAT-CMV3.2) all gave widespread expression of cGFP in A549 cells observed under an inverted fluorescence microscope (Fig. 4).

Inhibition of mRNA of the NEDD9 gene after transfection. A logarithmic chart of the corresponding concentrations according to the cycle threshold value $(\mathrm{Ct})$ of GAPDH and 

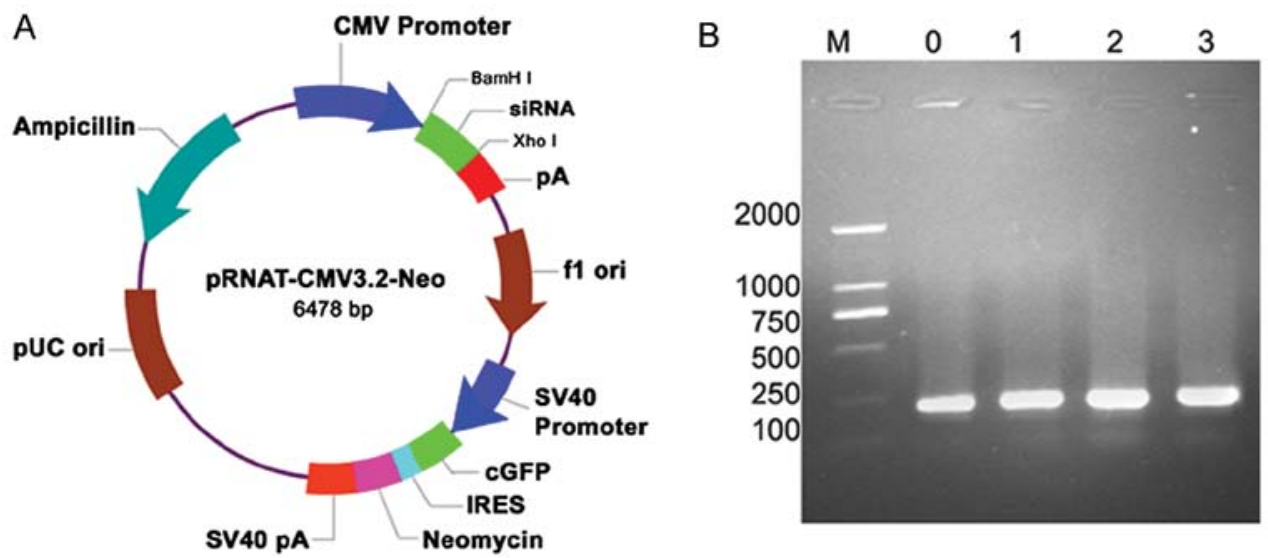

Figure 2. (A) Plasmid icon of pRNAT-CMV3.2. (B) PCR identification of recombinant clones. M, DNA marker; lane 0, amplified product of pRNATCMV3.2-CO; lane 1, pRNAT-CMV3.2-NE159 positive recon; lane 2, pRNAT-CMV3.2-NE193 positive recon; lane 3, pRNAT-CMV3.2-NE648 positive recon.

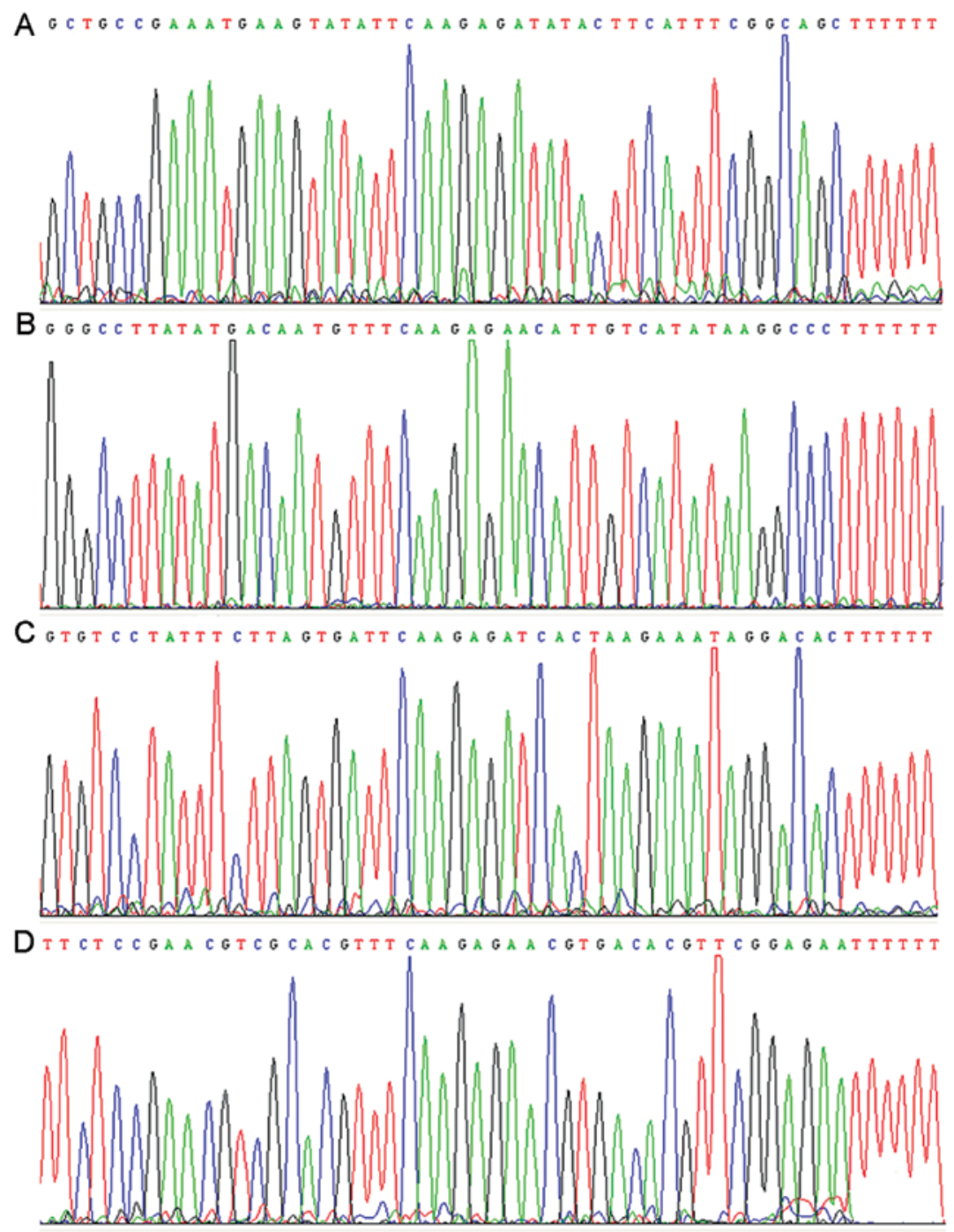

Figure 3. The insertion sequences completely matched with the designed sequences. (A) pRNAT-CMV3.2-NE159; (B) pRNAT-CMV3.2-NE193; (C) pRNATCMV3.2-NE648; (D) pRNAT-CMV3.2-C.

NEDD9 resulted in two straight lines. The relevant coefficients of GAPDH and NEDD9, i.e., straight lines indicated by real-time quantitative measurement, were -0.993 and -0.999 , respectively, and the gradients were both -3.24 . According to the formula $\mathrm{E}=10^{-1 / \mathrm{s}}-1$, where $\mathrm{E}$ is the amplification efficiency and $\mathrm{S}$ is the rate of standard curve; the amplification efficiency 
Table II. Relative expression of NEDD9.

\begin{tabular}{lccc}
\hline Group & $\Delta \mathrm{Ct}_{\text {mean }}$ & $\Delta \Delta \mathrm{Ct}$ & Fold (mean $\pm \mathrm{SD})$ \\
\hline Blank & 7.166 & 0 & 1 \\
pRNAT-CMV3.2 & 7.752 & 0.586 & $0.67 \pm 0.05^{\mathrm{a}}$ \\
pRNAT-CMV3.2-C & 7.763 & 0.597 & $0.68 \pm 0.17^{\mathrm{a}}$ \\
pRNAT-CMV3.2-N159 & 9.505 & 2.339 & $0.19 \pm 0.02^{\mathrm{b}, \mathrm{c}}$ \\
pRNAT-CMV3.2-N193 & 8.915 & 1.749 & $0.30 \pm 0.03^{\mathrm{b}, \mathrm{c}}$ \\
pRNAT-CMV3.2-N648 & 10.185 & 3.019 & $0.11 \pm 0.01^{\mathrm{b}, \mathrm{c}}$ \\
\hline
\end{tabular}

Fold $=2^{-\Delta \Delta \mathrm{Ct}} \cdot \Delta \Delta \mathrm{Ct}=\left(\mathrm{Ct}_{\mathrm{NEDD} 9}-\mathrm{Ct}_{\mathrm{GAPDH}}\right)_{\mathrm{pRNAT}-\mathrm{CMV} 3.2-\text {-siRNA }}-\left(\mathrm{Ct}_{\mathrm{NEDD} 9}-\mathrm{Ct}_{\mathrm{GAPDH}}\right)_{\text {blank }} \cdot{ }^{\text {a }}$ Two control groups, $\mathrm{P}>0.05 ;{ }^{\mathrm{b}}$ three siRNA groups, $\mathrm{P}>0.05$; cpRNAT-CMV3.2-N159, pRNAT-CMV3.2-N193, pRNAT-CMV3.2-N648 groups compared to the other groups, $\mathrm{P}<0.01$.
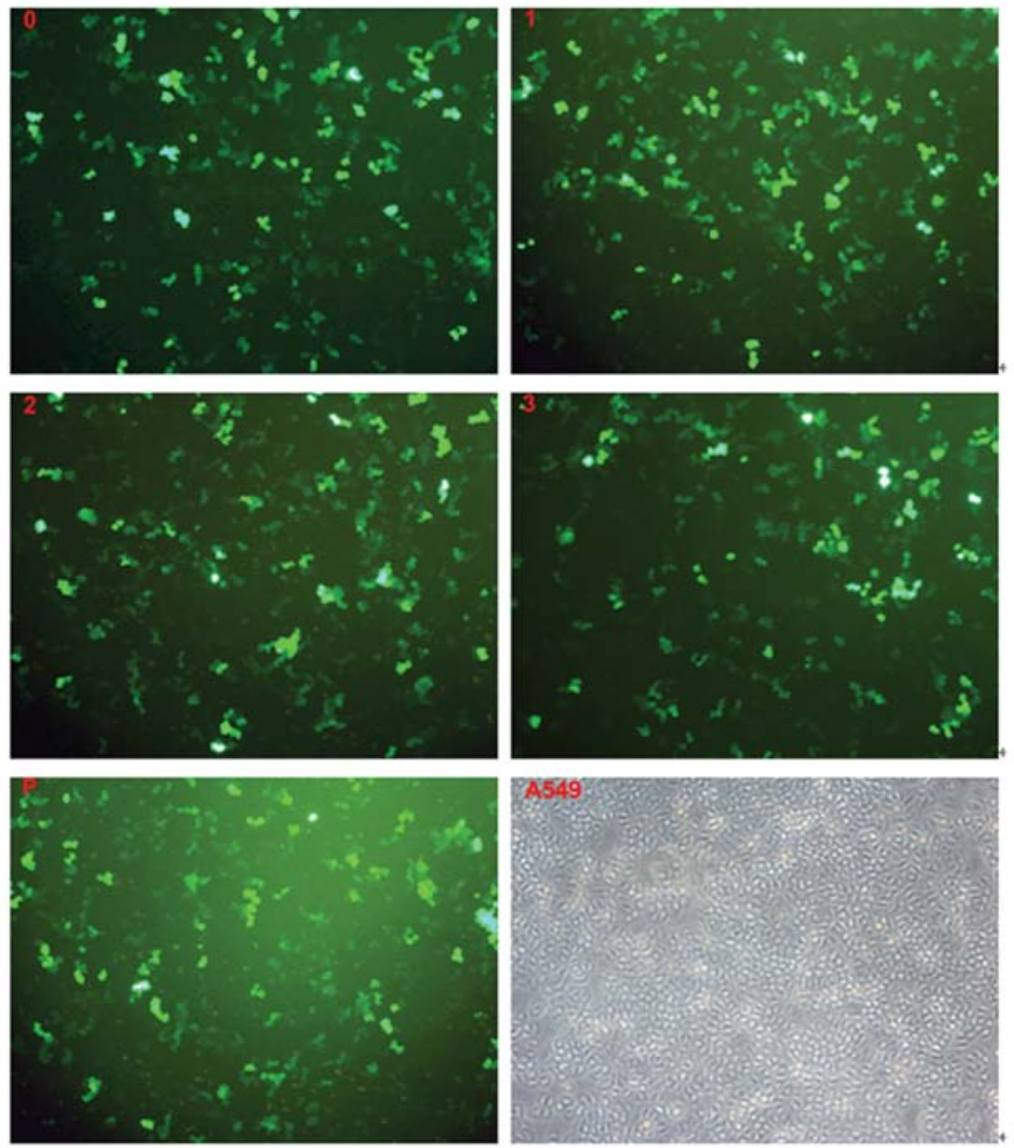

Figure 4. cGFP expression in A549 cells $48 \mathrm{~h}$ after transfection. DNA transfection efficiency after $48 \mathrm{~h}$ with Lipofectamine 2000. 0, Unrelated siRNA group; 1 , NE159 siRNA group; 2, NE193 siRNA group; 3, NE648 siRNA group; P, empty vector plasmid group. All slides are shown at the original magnification, $\mathrm{x} 100$.

of the two genes was $100 \%$. The $\mathrm{Ct}$ value for the plasmid pRNAT-CMV3.2-NE648 was lower in the intervention group compared with the blank group, and the rate of inhibition after correction was $86 \%$, which was significantly greater than other suppression rates $(\mathrm{P}<0.05)$ (Table II).

Inhibition of NEDD9 protein expression after transfection. Western blot analysis indicated that the experimental groups had low levels of NEDD9 protein expression while the control group had high levels of expression. There were no significant

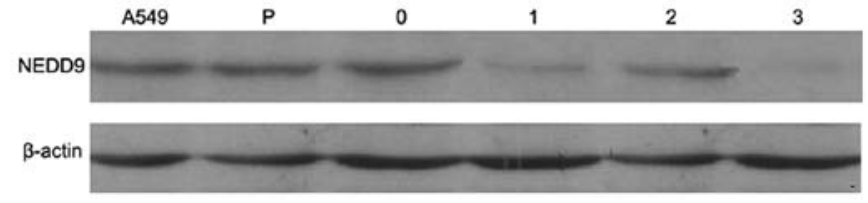

Figure 5. Western blotting for NEDD9 protein expression in A549 cells of the different groups. Lane A549, untransfected A549 cells; lane P, blank vector as control; lane 0 , unrelated siRNA as control; lane 1, transfected with pRNAT-CMV3.2-NE159; lane 2, transfected with pRNAT-CMV3.2-NE193; lane 3, transfected with pRNAT-CMV3.2-NE648. 
differences in $\beta$-actin protein expression between the six groups (Fig. 5).

\section{Discussion}

NEDD9 is a member of the CAS (Crk-associated substrate) protein family. Although it lacks any known enzymatic function, it contains many functional modules for protein interaction, leading to its classification as a scaffolding protein. Because it appears to lack catalytic activity, NEDD9 is not immediately promising as a target for directed drug development, unless it is through agents intended to disrupt protein-protein interactions, or through an siRNA-based approach to deplete NEDD9 levels globally.

siRNA-mediated knockdown of NEDD9 reduces the number of cells undergoing mitosis, and leads to cleavage furrow regression and multinucleation $(13,14)$. If NEDD9directed drugs or siRNAs should be developed, it is encouraging that genetic NEDD9-knockout animals exhibit relatively limited defects, as this implies that the loss of NEDD9 is well tolerated (15).

As a tool for gene therapy, RNAi technology has been applied to a number of diseases in medical research. For example, Lin et al (16) downregulated cancer-related gene expression by RNAi in human melanoma cells showing that the knockdown of gene expression significantly suppressed the abnormal proliferation of melanoma cells. Wang et al (17) successfully downregulated the mRNA and protein expression of Bcl-2 in breast cancer MCF27 cells by RNAi, revealing the valuable application of RNAi technology in basic research.

In this study, we designed and synthesized specific siRNA sequences targeting NEDD9 and successfully constructed four vectors, pRNAT-CMV3.2-N159, pRNAT-CMV3.2-N193, pRNAT-CMV3.2-N648 and pRNAT-CMV3.2-C. The human cytomegalovirus (CMV) promoter is one of the strongest promoters described. Based on the RNA polymerase II system, the CMV promoter drives higher-level constitutive expression of genes in a greater variety of mammalian cell lines compared with RNA polymerase III-based promoters such as U6 and $\mathrm{H} 1$. In this vector, the CMV promoter drives the expression of siRNA and a SV40 promoter drives the expression of the resistance gene. siRNA cassettes can be easily inserted into the vectors between the BamHI and XhoI sites. This vector also carries cGFP (coral GFP) for convenient tracking of transfection efficiency. FQ-PCR and western blotting showed substantially decreased mRNA and protein expression of the NEDD9 gene in the transfected cells, compared with the control group.

In conclusion, we designed a NEDD9 siRNA-expressing plasmid and showed that the siRNA expression vector inhibited the expression of NEDD9 in A549 cells. This study has laid the foundation for further therapeutic study of NEDD9 inhibition in lung adenocarcinoma.

\section{Acknowledgements}

The authors are grateful to all staff at the study centre who contributed to this study.

\section{References}

1. Law SF, Estojak J, Wang B, et al: Human enhancer of filamentation 1 (HEF1/NEDD9/CAS-L), a novel p130Cas-like docking protein, associates with FAK, and induces pseudohyphal growth in yeast. Mol Cell Biol 16: 3327-3337, 1996.

2. Minegishi M, Tachibana K, Sato T, et al: Structure and function of Cas-L, a 105-kD Crk-associated substrate-related protein that is involved in beta-1 integrin-mediated signaling in lymphocytes. J Exp Med 184: 1365-1375, 1996

3. Singh M, Cowell L, Seo S, et al: Molecular basis for HEF1/ NEDD9/Cas-L action as a multifunctional co-ordinator of invasion, apoptosis and cell cycle. Cell Biochem Biophys 48: 54-72, 2007.

4. Minn AJ, Gupta GP, Siegel PM, et al: Genes that mediate breast cancer metastasis to lung. Nature 436: 518-524, 2005.

5. Natarajan M, Stewart JE, Golemis EA, et al: HEF1 is a necessary and specific downstream effector of FAK that promotes the migration of glioblastoma cells. Oncogene 25: 1721-1732, 2006.

6. Kim M, Gans JD, Nogueira C, et al: Comparative oncogenomics identifies NEDD9 as a melanoma metastasis gene. Cell 125: 1269-1281, 2006

7. Jemal A, Bray F, Center MM, et al: Global cancer statistics. CA Cancer J Clin 61: 69-90, 2011.

8. Chang JX, Gao F, Zhang GJ, et al: Expression and clinical significance of NEDD9 in lung tissues. Med Oncol: March 24, 2012 (Epub ahead of print). doi: 10.1007/s12032-012-0213-0, 2012.

9. Law SF, Zhang YZ, Klein-Szanto AJ, et al: Cell cycle-regulated processing of HEF1 to multiple protein forms differentially targeted to multiple subcellular compartments. Mol Cell Biol 18: 3540-3551, 1998.

10. Ryu M, Kinoshita T, Konishi M, et al: Segmental resection of the duodenum including the papilla of Vater for focal cancer in adenoma. Hepatogastroenterology 43: 835-838, 1996.

11. Lowell JA, Rossi RL, Munson JL, et al: Primary adenocarcinoma of third and fourth portions of duodenum. Arch Surg 127: $557-560,1992$.

12. Kaklamanos IG, Bathe OF, Franceschi D, et al: Extent of resection in the management of duodenal adenocarcinoma. Am J Surg 179: 37-41, 2000.

13. Pugacheva EN and Golemis EA: The focal adhesion scaffolding protein HEF1 regulates activation of the Aurora-A and Nek2 kinases at the centrosome. Nat Cell Biol 7: 937-946, 2005.

14. Dadke D, Jarnik M, Pugacheva EN, et al: Deregulation of HEF1 impairs M-phase progression by disrupting the RhoA activation cycle. Mol Biol Cell 17: 1204-1217, 2006.

15. Seo S, Asai T, Saito T, et al: Crk-associated substrate lymphocyte type is required for lymphocyte trafficking and marginal zone B cell maintenance. J Immunol 175: 3492-3501, 2005.

16. Yin JQ and Wan Y: RNA-mediated gene regulation system: Now and the future. Int J Mol Med 10: 355-365, 2002.

17. Wang YH, Liu S, Zhang G, et al: Knockdown of c-Myc expression by RNAi inhibits MCF-7 breast tumor cells growth in vitro and in vivo. Breast Cancer Res 7: 220-228, 2005. 